\title{
A Dual Self Model of Impulse Control ${ }^{1}$
}

\author{
Drew Fudenberg and David K. Levine ${ }^{2}$
}

First Version: August 5, 2004

This Version: February 26, 2005

\begin{abstract}
We propose that a simple "dual-self" model gives a unified explanation for several empirical regularities, including the apparent time-inconsistency that has motivated models of hyperbolic discounting and Rabin's paradox of risk aversion in the large and small. The model also implies that self-control costs imply excess delay, as in the O'Donoghue and Rabin models of hyperbolic utility, and it explains experimental evidence that increased cognitive load makes temptations harder to resist. Finally, the reduced form of the base version of our model is consistent with the Gul-Pesendorfer axioms.
\end{abstract}

\footnotetext{
${ }^{1}$ We thank David Laibson and Matt Rabin for many years of instructive conversations on related topics, Stefano DellaVigna and Ulrike Malmendier for helpful discussion of their research, and Drazen Prelec and Antonio Rangel for detailed comments on an earlier draft. We are grateful to NSF grants SES-01-12018, SES-03-14713, and SES-04-26199 for financial support.

${ }^{2}$ Department of Economics Harvard University, and UCLA/Federal Reserve Bank of Minneapolis.
} 
"The idea of self-control is paradoxical unless it is assumed that the psyche contains more than one energy system, and that these energy systems have some degree of independence from each other."

(McIntosh [1969])

\section{Introduction}

This paper argues that a simple "dual-self" model gives a unified explanation for a number of empirical regularities. This includes the apparent time inconsistency that has motivated economists' models of hyperbolic discounting: Faced with a choice between consuming some quantity today and a greater quantity tomorrow, some people will choose to consume the lesser quantity today. However, when these same individuals are faced with the choice between the same relative quantities a year from now and a year and a day from now, they choose to consume the greater quantity a year and a day from now. ${ }^{3}$ The second regularity is Rabin's [2000] paradox of risk aversion in the large and small. The paradox is that the risk aversion experimental subjects show to very small gambles implies hugely unrealistic willingness to reject large but favorable gambles. In addition, the model explains the effect of cognitive load on self-control that is noted by Shiv and Fedorkin, and it predicts that increased costs of self-control lead to increased delay in stationary stopping-time problems, as in O’Donoghue and Rabin [2001].

Our theory proposes that many sorts of decision problems should be viewed as a game between a sequence of short-run impulsive selves and a long-run patient self. This is consistent with recent evidence from MRI studies such as McClure, Laibson, Loewenstein, and Cohen [2004] that suggest that short-term impulsive behavior is associated with different areas of the brain than long-term planned behavior. ${ }^{4}$ The

\footnotetext{
${ }^{3}$ The economics literature on hyperbolic discounting, following Strotz [1955] and Laibson [1997], uses the now-familiar $(\beta, \delta)$ form. This is called "quasi-hyperbolic discounting" in the psychology literature to distinguish it from the discounting function $f(t)=(1+\alpha t)^{\beta / \alpha}$, which actually is hyperbolic. See Prelec [2004] for a characterization of these functions in terms of "deceasing impatience."

${ }^{4}$ They say "Parts of the limbic system associated with the midbrain dopamine system... are preferentially activated by decisions involving immediately available rewards. In contrast, regions of the lateral prefrontal cortex and posterior parietal cortex are engaged uniformly by intertemporal choices irrespective of delay. Furthermore, the relative engagement of the two systems is directly associated with subjects' choices, with greater relative fronto-parietal activity when subjects choose longer term options."
} 
findings of this research are reinforced also by introspection - we are all aware of the internal conflict when our "rational self" is faced with short-term indulgences that lead to bad long-term consequences. We argue that our theory explains a broad range of behavioral anomalies, and that it is a better fit for the modular structure of the brain than the hyperbolic model, which posits a game between multiple "selves," one in each period. ${ }^{5}$ Moreover, the dual-selves model is analytically simpler than the hyperbolic discounting model: it always yields a unique equilibrium that can be calculated as the solution to a decision problem. The only model of hyperbolic preferences we know of that has similar properties is the Harris and Laibson [2004] model of instantaneous gratification in a consumption-savings problem. That model seems to us to be more complicated and specialized than our own.

In our model, the patient long-run self and a sequence of myopic short-run selves share the same preferences over stage-game outcomes; they differ only in how they regard the future. Specifically, we imagine that the short-run myopic self has "basepreferences" in the stage game that depend only on the outcome in the current stage. That is, the short-run players are completely myopic. ${ }^{6}$

The stage game is played in two phases. In the first phase, the long-run self chooses the utility function of the myopic self. At some reduction in utility (for both selves - who share the same stage game utility function) the long-run self can choose preferences other than the "base preferences. In the second phase of the stage game, after the short-run player preferences have been chosen, the short-run player takes the final decision. It is important that we do not allow the long-run self to precommit for the entire dynamic game. Instead, she begins each stage game facing the choice of which preferences to give the myopic self - or equivalently, how much self-control to exert. Note also that while the hyperbolic discounting model emphasizes the conflict between present and future selves, we emphasize that the long-run self has the same stage game

\footnotetext{
${ }^{5}$ At the same time, we recognize that our model is only a very loose approximation of the brain's structure, as recent studies suggest both that reward-related information may be processed in many different brain regions, and that the links between these region are more complex than the top-down control assumed in our model. See for example O'Doherty [2004] and Platt and Glimcher [1999].

${ }^{6}$ This is a very stark assumption, but it leads to a much simpler model, and may be a reasonable approximation in some cases of interest. The conclusion discusses the complications introduced by forward-looking "short run selves."
} 
preferences as the short-run self, and so wishes to serve the interests of future short-term selves.

Games with long run versus short run players are relatively simple to analyze. This particular class is especially simple. Imposing a minimal perfection requirement that the short-run self must always play a best response, the long-run self implicitly controls the short-run self, albeit at some cost. Equilibria of this game are equivalent to the solution to an optimization problem. In this respect, the long-run versus short-run player model is more conservative than hyperbolic discounting, preserving many of the methods and insights of existing theory, as well as delivering strong predictions about behavior.

Our model is similar in spirit to that of Thaler and Shefrin [1981] (from whom we have taken the McIntosh quotation at the start of the paper.) Like them, we view our model as "providing a simple extension of orthodox models that permits [self-control behavior] to be viewed as rational." One difference is that their model is defined only for the consumption-savings problem we study in section 3, while we develop a more general model that can be applied to other situations. Also, we work with more precise specifications of the costs of self-control, and show how to reduce the game between the selves to a single decision problem. This makes the model analytically tractable, and enables us to make more precise predictions. Independent work by O'Donoghue and Lowenstein [2004] describes a similar but more general model, with less focus on tractability and applications. Benabou and Pycia [2002] analyze a one-period model where the long-run self and the short-run self compete for control by expending resources, with the probability that a given self takes control equal to its share of the total expenditure. Bernheim and Rangel [2004], and Benahib and Bisin [2004] consider multiperiod models where a long-run self is only sometimes in control, either because it is unable to take control (Bernheim and Rangel) or chooses not to do so (Benahib and Bisin.) We discuss these papers further in the conclusion. Section 5 discusses Miao [2004], who applies the dual-self model to a variant of the waiting-time problem we analyze in that section.

Although our point of departure is different, the reduced form of the dual self model is closely connected to the dynamic model of Gul and Pesendorfer [2002]. They consider a single player who has preferences over choice sets that includes the desire to limit the available alternatives. Under a particular set of axioms, they show that the 
decision process can be represented by a utility function with a cost of self-control that is closely connected to ours. Although the reduced form of the model leads to the similar decision problem, we have a concrete interpretation of preferences in terms of those of a myopic self, and as a result are able to bring both introspective and physiological evidence to bear on what those preferences might be.

The dual self model predicts the same behavioral anomalies that motivated the hyperbolic discounting model, albeit through a different mechanism. When dealing with decisions that effect only future options, the short-run self is indifferent, hence can be manipulated by the long run self at minimal cost. The long-run self, then, has two different sorts of mechanisms through which to change the behavior of future short-run selves. She can intervene directly in a future stage game by choosing an appropriate utility function, but to do so requires a substantial utility cost. Alternatively, in some settings it may be possible for the current short-run self to limit the alternatives available to the future short-run selves; manipulating these decisions has negligible cost. For example, in the classical choice problem that gave rise to the theory of hyperbolic discounting, faced with a decision between consuming something today or more tomorrow, the long-run decision maker may prefer to consume more tomorrow. But to manipulate the current short-run self into making that decision may be more costly than it is worth. On the other hand, when faced with making a commitment to the same decision involving future dates, the cost of manipulating the decision of the current short run self is negligible, since the current short-run self does not care about the future. ${ }^{7}$

For a less trivial application, we examine a simple one-person savings problem. We show that if the short-run self has access to all available wealth, the savings rate is reduced to keep the cost of self-control low. On the other hand, when wealth is kept in a bank account, and the short-run self that withdraws the money is different from the shortrun self who (at a later time) spends the money, savings are exactly those predicted in the

\footnotetext{
${ }^{7}$ We should point out that this discussion implicitly supposes that the short-run self is directly motivated by money, even though the only real consequence of earning money is the future consumption that it brings. This is consistent with evidence (such as Pavlov's bell) that the impulsive short-run self responds to learned behavioral cues in addition to direct stimulus. Modern physiological research is making progress in identifying some of the brain chemistry that reflects the response to these stimuli, see, for example, Haruno et al [2004]. Camerer, Lowenstein and Prelec [2000] say that "roughly speaking, it appears that similar brain circuitry-dopaminergic neurons in the midbrain - is active for a wide variety of rewarding experiences (including) money rewards." The conclusion speculates about some possible extensions of our model to learned cues.
} 
absence of self-control costs. However, the dual self model predicts that the propensity to spend out of unanticipated cash receipts is greater than out of unanticipated bank-account receipts. In particular, a sufficiently small unanticipated cash receipt will be spent in its entirety, and so winnings from sufficiently small cash gambles are evaluated by the shortrun self's preferences, which are over consumption. These preferences are more riskaverse than the preferences over long-term consumption that are used to evaluate large gambles, so this "cash effect" provides an explanation of Rabin's [2000] paradox of risk aversion in the small and in the large. ${ }^{8}$

We also apply the dual-self model to the study of procrastination and delay in a stationary stopping-time environment that is very similar to that of O'Donoghue and Rabin [2001]. Like them, we find that self-control costs lead to longer delays, but our model yields a unique prediction, in contrast to their finding of multiple equilibria. Our model also suggests some qualifications to the interpretations that DellaVigna and Malmendier [2003] give to their data on health-club memberships.

\section{The Model}

Time is discrete and potentially unbounded, $t=1,2, \ldots$ There is a fixed, timeand history invariant set of actions $A$ for the short-run selves; this is assumed to be a compact subset of Euclidean space. ${ }^{9}$ There is also a measure space $Y$ of states, and a set $R$ of self-control actions for the long-run self; $R$ is a compact convex subset of Euclidean space. The point $0 \in R$ is taken to mean that no self-control is used. A finite history of play $h \in H$ consists of the past states and actions, $h=\left(y_{1}, a_{1}, r_{1}, \ldots, y_{t}, a_{t}, r_{t}\right)$ or the null history 0 . The length of the history is denoted by $t(h)$, the final state in $h$ by $y(h)$. There is an initial state $y_{1}$. The probability distribution over states at time $t+1$ depends on the time- $t$ state and action $y_{t}, a_{t}$ according to the exogenous probability measure $\mu(y, a)$; the long-run self's action $r$ has no effect on the future state. ${ }^{10}$ The game is between the longrun self, whose pure strategies are maps from histories and the current game to self-

\footnotetext{
${ }^{8}$ As we explain below, the cash effect has the same impact in the hyperbolic discounting mode. Note also that this result is in the opposite direction from those of Gul and Pesendorfer [2002, 2004], who do not consider environments with mechanisms such as banks that substitute for self-control.

${ }^{9}$ This assumption is a modeling convenience, but it can be relaxed to allow for history-dependent action sets.

${ }^{10}$ This is another simplifying assumption; when it is dropped the long-run player's maximization problem becomes more complicated.
} 
control actions $\sigma_{L R}: H \times Y \rightarrow R$, and the sequence of short-run selves. Each short-run self plays in only one period, and observes the self-control action chosen by the long-run self prior to moving. Denote by $H_{t}$ the set of $t$-length histories $H_{t}$. A strategy from the time- $t$ short-run self is a map $\sigma_{t}: H_{t} \times Y \times R \rightarrow A$; we denote the collection of all of these strategies by $\sigma_{S R}$. The strategies together with the measure $\mu$ give rise to a measure $\pi_{t}$ over histories of length $t$.

The utility of the short-run self is given by $u(y, r, a)$ : the long-run player's current self-control action influences the short-run player's payoff. The utility of the long-run self is given by

$$
U=\sum_{t=1}^{\infty} \delta^{t-1} \int u(y, r, a) d \pi_{t}(y(h))
$$

Because $r$ has no impact om the evolution of the state variable $y$, the model supposes that all interactions with the outside world are handled by the short-run self. ${ }^{11}$ The long-term self can influence these interactions by changes in $r$; this will be particularly easy when the actions available have no immediate consequences so that the short-term self is indifferent. Physiologically, we imagine that self-control corresponds to the release of chemicals that determine "mood" and other variables relevant to the preferences of the impulsive myopic self.

In this formulation, the self-control cost (that is, the difference between $u(y, r, a)$ and $u(y, 0, a))$ is borne by both selves. However, since the short-run self cannot influence that cost, all that matters is the influence of self-control on the marginal incentives of the short-run self and thus on its decisions.

Since each move begins a proper subgame, the strategies are a subgame perfect equilibrium if each self's strategy is optimal following every history, and the short-run self's strategy is also optimal following the move of the long-run self.

Assumption 1 (Costly Self-Control): If $r \neq 0$ then $u(y, r, a)<u(y, 0, a)$.

\footnotetext{
11 Thus the short-term self in our model represents a combination of a short-term reward assessor and a "doer."
} 
Assumption 2 (Unlimited Self-Control): For all y, a there exists $r$ such that for all $a^{\prime}$, $u(y, r, a) \geq u\left(y, r, a^{\prime}\right)$.

Under the assumptions that self-control is costly and unlimited, we may define the cost of self-control

$$
C(y, a) \equiv u(y, 0, a)-\sup _{\{r \mid u(y, r, a) \geq u(y, r,)\}} u(y, r, a)
$$

Assumption 3 (Continuity): $u(y, r, a)$ is continuous in $r, a$.

This assures that the supremum in the definition of $C$ can be replaced with a maximum.

Assumption 4 (Limited Indifference): for all $a^{\prime} \neq a$, if $u(y, r, a) \geq u\left(y, r, a^{\prime}\right)$ then there exists a sequence $r^{n} \rightarrow r$ such that $u\left(y, r^{n}, a\right)>u\left(y, r^{n}, a^{\prime}\right)$.

This means that when the short-run self is indifferent, the long-run self can break the tie for negligible cost.

Notice that from Assumptions 1 and 2, if $a=\arg \max _{a^{\prime}}\left(u\left(y, 0, a^{\prime}\right)\right)$ then $C(y, a)=0$, and $C\left(y, a^{\prime}\right)>0$ for $a^{\prime} \neq a$. In addition by Assumption 3, $C(y, a)$ is continuous in $a$. Conversely, if we have given functions $u(y, 0, a)$ and $C(y, a)$ satisfying these properties, then we can take $R=A$ and construct

$$
u(y, r, a)= \begin{cases}u(y, 0, r)-C(y, r)-\|r-a\| \quad a \mid u(y, 0, a) \geq u(y, 0, r) \\ u(y, 0, a)-C(y, r)-\|r-a\| \quad a \mid u(y, 0, a)<u(y, 0, r)\end{cases}
$$

which gives rise to the target cost of self-control function, while satisfying Assumptions $1-4$.

By way of contrast, we consider also the following reduced form optimization problem, of choosing a strategy from histories and states to actions $\sigma_{S C}: H \times Y \rightarrow A$ to maximize the objective function

$$
\sum_{t=1}^{\infty} \delta^{t-1} \int[u(y, 0, a)-C(y, a)] d \pi_{t}(y(h)) .
$$

Theorem 1 (Equivalence of Subgame Perfection to the Reduced Form): A subgame perfect equilibrium exists if and only if a solution to the reduced form problem exists; if a 
solution exists then for every optimal $\sigma_{S C}$ there are equilibrium strategies $\sigma_{L R}, \sigma_{S R}$ such that $\sigma_{S C}=\sigma_{L R} \circ \sigma_{S R}$ and vice versa.

Remark: We have not imposed sufficient assumptions on $Y$ and $\mu$ to guarantee the existence of a solution to the optimization problem. If $Y$ is finite, it is well known that this problem has a solution; however we wish to examine cases where $Y$ is infinite, and although in our examples existence of a solution is unproblematic, it is complicated to give general conditions guaranteeing the existence of an optimum in the infinite case.

Finally, we wish to consider a tightly parameterized functional form for the cost of self-control, namely that it is proportional to the difference in utility between the best available action and that actually taken.

Assumption 5 (Linear Self-Control Cost): $C(y, a)=\gamma\left[\max _{a^{\prime}} u\left(y, 0, a^{\prime}\right)-u(y, 0, a)\right]$, so that

$$
\begin{aligned}
U= & \sum_{t=1}^{\infty} \delta^{t-1} \int[u(y, 0, a)-C(y, a)] d \pi_{t}(y(h)) \\
& \left.=\sum_{t=1}^{\infty} \delta^{t-1} \int\left[(1+\gamma) u(y, 0, a)-\gamma \max _{a^{\prime}} u\left(y, 0, a^{\prime}\right)\right)\right] d \pi_{t}(y(h))
\end{aligned}
$$

Notice that the introduction of self-control costs into the optimization problem means that introducing more options can make the agent worse off. However, under Assumption 5, although improving the best available alternative can lower utility, it does not change the marginal cost of self-control. In this sense, Assumption 5 is conservative, maintaining as much of the standard model as is consistent with an interesting theory of self-control. It is also important to note that self-control costs that satisfy Assumption 5 are shown by Gul and Pesendorfer [2001, 2002] to satisfy their axioms. ${ }^{12}$ Assumption 5 is also analytically convenient. On the other hand, Section 6 argues that some evidence is more consistent with strictly convex costs of control.

\section{A Simple Savings Model}

To start, consider the simple case of an infinite-lived consumer making a savings decision. The state $y \in \Re_{+}$represents wealth, which may be divided between

\footnotetext{
${ }^{12}$ Benabou and Pycia [2002] make the same point about the one-period version of this model.
} 
consumption and savings according to the action $a \in[0,1]$ representing the savings rate. Borrowing is not allowed. Savings are invested in an asset that returns wealth $y_{t+1}=R a_{t} y_{t}$ next period, there is no other source of income. ${ }^{13}$

In each period of time, the base preference of the short-run self has logarithmic utility, $^{14}$

$$
u(y, 0, a)=\log ((1-a) y)
$$

where we define $\log (0)=-\infty$.

The short-run self wishes to spend all available wealth on consumption. We assume a separable cost of self-control, so

$$
\begin{aligned}
& \left.C(y, a)=\gamma\left[\max _{a^{\prime}} u\left(y, 0, a^{\prime}\right)-u(y, 0, a)\right]=\gamma(\log (y)-\log (1-a) y)\right) \\
& =-\gamma \log (1-a) .
\end{aligned}
$$

The reduced form for the long-run self has preferences

$$
\begin{aligned}
U & =\sum_{t=1}^{\infty} \delta^{t-1} \int\left[u\left(y_{t}, 0, a_{t}\right)-C\left(y_{t}, a_{t}\right)\right] d \pi_{t}(y(h)) \\
& =\sum_{t=1}^{\infty} \delta^{t-1}\left[(1+\gamma) \log \left(\left(1-a_{t}\right) y_{t}\right)-\gamma \log \left(y_{t}\right)\right]
\end{aligned}
$$

The long-run self's problem is thus to maximize this function subject to the wealth equation $y_{t}=R a_{t-1} y_{t-1}$. It is shown in the Appendix that there is a solution, and that the solution has a constant savings rate strictly between zero and one. ${ }^{15}$ Thus we compute present value utility for constant savings rates, and maximize

\footnotetext{
${ }^{13}$ Because we take the short-run self's action to be the savings rate and not total savings, the feasible actions $A$ are independent of the long-run self's actions. Note also that since the short-run self makes all consumption/savings decisions, the model satisfies our requirement that the evolution of the state depends only on the actions of the short-run self.

${ }^{14}$ The Appendix presents the extension to CRRA form of which logarithmic utility is a special case. We show that the problem has a solution, and that the solution is characterized by a first-order condition. We also solve the banking model of the next section with CRRA preferences. Because these preferences have an extra parameter of flexibility, they seem likely to better fit field data; we present the logarithmic case in the text to get across the main ideas.

15 As we have written the problem, with the savings rate as the control, the state evolution equation is not concave; if we change variables so that the control is the absolute level of consumption $c_{t}$, the state evolution equation is linear but the per-period payoff becomes $(1+\gamma) \log \left(c_{t}\right)-\gamma \log \left(y_{t}\right)$, which is not
} 


$$
\begin{aligned}
& \begin{array}{l}
U=\sum_{t=1}^{\infty} \delta^{t-1}\left[(1+\gamma)\left(\log (1-a)+(t-1) \log R a+\ln y_{0}\right)-\gamma(t-1) \log (R a)-\gamma \log \left(y_{0}\right)\right] \\
\quad=\sum_{t=1}^{\infty} \delta^{t-1}\left[(1+\gamma)(\log (1-a))+(t-1) \log (R a)+\log \left(y_{0}\right)\right]
\end{array} \\
& \text { using } \sum_{t=1}^{\infty} t \delta^{t}=\frac{\delta}{(1-\delta)^{2}} \text {, this simplifies to }
\end{aligned}
$$

$$
\begin{aligned}
U & =\frac{\left[(1+\gamma)(\log (1-a))+\log \left(y_{0}\right)\right]}{(1-\delta)}+\frac{\log (R a)}{(1-\delta)^{2}}-\frac{\log (R a)}{(1-\delta)} \\
& =\frac{\left[(1+\gamma)(\log (1-a))+\log \left(y_{0}\right)\right]}{(1-\delta)}+\frac{\delta \log (R a)}{(1-\delta)^{2}}
\end{aligned}
$$

To find the constant savings rate that maximizes $U$, we differentiate (1) to find

SO

$$
\begin{gathered}
d U / d a=\frac{-(1+\gamma)}{(1-a)(1-\delta)}+\frac{1}{a(1-\delta)^{2}}-\frac{1}{a(1-\delta)}, \\
a=\frac{\delta}{1+\gamma-\delta \gamma}
\end{gathered}
$$

Since the solution must be interior, it must satisfy the first-order condition, and since there is a unique solution to the first order condition, this is the optimum

The comparative statics are immediate and intuitive: As $\gamma$ increases, so selfcontrol becomes more costly, the savings rate is reduced, to avoid the cost of self-control. As the long-run player becomes more patient, (as $\delta$ increases) this cost of future self control becomes more important, so the effect of $\gamma$ increases, which tends to increase the difference between the savings rate at a fixed $\gamma$ and that at $\gamma=0$. (In particular, $\gamma$ is irrelevant when $\delta=0$, as the savings rate is 0 with or without costs of self-control.) However, increasing $\delta$ also increases the savings rate for any fixed $\gamma$, as is the case

concave in the state if $\gamma>0$. For this reason our proof technique does not rely on concavity. We can extend the conclusion that savings are a constant fraction of wealth to the case where asset returns $\tilde{R}$ are stochastic and i.i.d. provided that there is probability 0 of 0 gross return. In the more general CRRA case studied in the Appendix, the solution given there remains unchanged provided we define $R^{1-\rho}=E\left(\tilde{R}^{1-\rho}\right)$. 
when $\gamma=0$ and there is no self-control problem. This latter effect dominates, as total saving increases.

Note that when $\gamma=0$, so there are no self-control costs, the optimum savings rate is $a^{*}=\delta$. In this case the agent's lifetime utility as a function of initial wealth $y_{0}$ is

$$
\frac{\left[\log (1-\delta)+\log \left(y_{0}\right)\right]}{(1-\delta)}+\frac{\delta \log (R \delta)}{(1-\delta)^{2}}
$$

we use this fact in the following section.

To summarize, our model has a constant savings rate for both logarithmic and CRRA utility, and the savings rate is the solution to a first-order condition; the solution is particularly simply in the case of logarithmic utility. In contrast, as Harris and Laibson [2004] emphasize, consumption need not be monotone in wealth in the usual discretetime hyperbolic model, even in a stationary infinite-horizon environment. Moreover, the hyperbolic model typically has multiple equilibria (Krusell and Smith [2000]), which complicates both its analysis and its empirical application.

In response, Harris and Laibson [2004] propose a continuous-time model of the consumption-savings problem, where the return on savings is a diffusion process. They show that the equilibrium is unique in the limit where individuals prefer gratification in the present discretely more than consumption in the only slightly delayed future. ${ }^{16}$ Moreover, in our case of constant return on assets, their results show that consumption is a constant fraction of wealth if the discount factor is sufficiently close to 1 . Thus the limit form of their model makes qualitatively similar predictions to ours; we feel that our approach is more general and more direct.

\section{Simple Banking Model}

In practice there are many ways of restraining the sort-run self besides the use of self-control: the obvious thing to do is make sure that the short-run self does not have access to resources that would represent a temptation. Here we consider the consequences

\footnotetext{
${ }^{16}$ To do this, they show that equilibrium is characterized by the solution of a single-agent problem, where the agent's utility function is derived from the shadow values in the original problem. When base preferences are CRRA, the only difference between the derived utility function and that of a "fully rational" agent (an exponential discounter) is that the agent gets a utility boost at zero wealth,
} 
of a simple model in which basic savings decisions are made in a context (the bank) where consumption temptations are not present. In the bank, the decision is made how much "pocket cash" to make available for spending when a consumption opportunity arises in the following period. Since savings decisions are made in the bank, with perfect foresight, the optimum without self-control can be implemented simply by rationing the short-run self. Thus the baseline, deterministic version of the model has an equilibrium equivalent to a model without a self-control problem. However, consumer's response to unanticipated cash receipts is quite different than that to anticipated receipts, or to unanticipated bank account receipts: the propensity to consume out of a small unanticipated cash receipt is $100 \%$, while the propensity to consume out of a similar amount of money received in the bank account (for example, a small capital gain on a stock) is small.

This wedge between the propensity to consume out of pocket cash and to consume out of bank cash has significant implications for "risk aversion in the large and small." Winnings from sufficiently small cash gambles are spent in their entirety, and so are evaluated by the short-run self's preferences, which are over consumption. When the stakes are large, self-restraint kicks in, part of the winnings will be saved and spread over the lifetime. This leads to less risk-averse preferences, so the model explains the paradox proposed by Rabin [2000]. Note that these results are in the opposite direction of Gul and Pesendorfer [2002, 2004], who do not consider environments with mechanisms such as banks that substitute for self-control.

The implication of the pocket versus bank cash model are very important in the interpretation of experimental results: in experiments the stakes are low, but individuals demonstrate substantial curvature in the utility function. Besides exhibiting risk aversion, when given the opportunity, for example, to engage in altruistic behavior, they generally do not make the minimum or maximum donation, but some amount in between. (Similar behavior is observed on the street: many people will make a positive donation to a homeless person, but few will empty their pockets of all cash.) If utility is viewed in terms of wealth, this type of behavior makes little sense, since the effect of a small donation on the marginal utility of wealth to either the donor or recipient is miniscule. Viewed in terms of pocket cash, which is the relevant point of comparison when there is 
a wedge due to the rationing of cash to the short-run self, this behavior makes perfect sense.

Formally, we augment the simple saving model by supposing that each period consists of two subperiods, the "bank" subperiod and the "nightclub" subperiod. During the "bank" subperiod, consumption is not possible, and wealth $y_{t}$ is divided between savings $s_{t}$, which remains in the bank, and cash $x_{t}$ which is carried to the nightclub. In the nightclub consumption $0 \leq c_{t} \leq x_{t}$ is determined, with $x_{t}-c_{t}$ returned to the bank at the end of the period. Wealth next period is just $y_{t+1}=R\left(s_{t}+x_{t}-c_{t}\right)$. The discount factor between the two consecutive nightclub periods (which is where consumption occurs) is $\delta$; preferences continue to have the logarithmic form. ${ }^{17}$ First, consider the perfect foresight problem in which savings are the only source of income. Since no consumption is possible at the bank, the long-run self gets to call the shots; and the longrun self can implement $a^{*}=\delta$, the optimum of the problem without self-control, simply by choosing pocket cash $x_{t}=\left(1-a^{*}\right) y_{t}$ to be the desired consumption. The short-run self will then spend all the pocket cash; because the optimum can be obtained without incurring self-control costs, the long-run self does not in fact wish to exert self-control at the nightclub.

Now we turn to the problem of stochastic cash receipts (or losses). That is, we suppose that at the nightclub in the first period there is a small probability the agent will be offered a choice between several lotteries. If the lotteries are themselves drawn in an i.i.d. fashion, this will also result in a stationary savings rate that is slightly different from the $a^{*}$ computed above, but if the probability that a non-trivial choice is drawn is small, the savings rate will be very close to $a^{*}$. We find it easier to consider the limit where the probability of drawing the gamble is zero.

For the agent to evaluate a lottery choice $\tilde{z}_{1}$, he needs to consider how he would behave conditional on each of its possible realizations $z_{1}$. The short-run self is constrained to consume $c_{1} \leq x_{1}+z_{1}$. Next period wealth is given by

$$
y_{2}=R\left(s_{1}+x_{1}+z_{1}-c_{1}\right)=R\left(y_{1}+z_{1}-c_{1}\right) \text {. }
$$

\footnotetext{
${ }^{17}$ The appendix provide the parallel computations for the CRRA case.
} 
The utility of the long-run self starting in period 2 is given by the solution of the problem without self control, as in equation (3):

$$
U_{2}\left(y_{2}\right)=\frac{1}{(1-\delta)}\left(\log (1-\delta)+\log \left(y_{2}\right)+\frac{\delta}{1-\delta} \log (R \delta)\right)
$$

The utility of both selves in the first period is $(1+\gamma) \ln \left(c_{1}\right)-\gamma \ln \left(x_{1}+z_{1}\right)$, and so the overall objective of the long-run self is to maximize

$$
\begin{aligned}
& (1+\gamma) \log \left(c_{1}\right)-\gamma \log \left(x_{1}+z_{1}\right) \\
& +\frac{\delta}{(1-\delta)}\left(\log (1-\delta)+\log \left(R\left(y_{1}+z_{1}-c_{1}\right)\right)+\frac{\delta}{1-\delta} \log (R \delta)\right)
\end{aligned}
$$

The first order condition for optimal consumption is

$$
\frac{1+\gamma}{c_{1}}=\frac{\delta}{(1-\delta)\left(y_{1}+z_{1}-c_{1}\right)}
$$

so

$$
c_{1} \delta=(1-\delta)\left(y_{1}+z_{1}-c_{1}\right)+\gamma(1-\delta)\left(y_{1}+z_{1}-c_{1}\right)
$$

and

$$
\begin{aligned}
c_{1} & =\frac{(1-\delta)(1+\gamma)\left(y_{1}+z_{1}\right)}{\delta+(1+\gamma)(1-\delta)}=\left(1-\frac{\delta}{\delta+(1+\gamma)(1-\delta)}\right)\left(y_{1}+z_{1}\right) \\
& \equiv(1-B)\left(y_{1}+z_{1}\right)
\end{aligned}
$$

Note that when $\gamma=0$, (5) simplifies to $c_{1}=(1-\delta)\left(y_{1}+z_{1}\right)$, as it should. If the solution $c_{1}$ satisfies the constraint $c_{1} \leq x_{1}+z_{1}$ it represents the optimum; otherwise the optimum is to consume all pocket cash, $c_{1}=x_{1}+z_{1}$. Because $x_{1}$ is the solution for $\gamma=0$, we know that $x_{1}=(1-\delta) y_{1}$. Thus $c_{1} \leq x_{1}+z_{1}$ if $z_{1}<z_{1}^{*}$, where the critical value of $z_{1}^{*}$ is derived from

$$
\left(1-\frac{\delta}{\delta+(1+\gamma)(1-\delta)}\right)\left(y_{1}+z_{1}^{*}\right)=(1-\delta) y_{1}+z_{1}
$$


This yields

$$
\begin{aligned}
& (1-B)\left(y_{1}+z_{1}^{*}=(1-\delta) y_{1}+z_{1}^{*}\right. \\
& z_{1}^{*}=(\delta / B-1) y_{1} \\
& z_{1}^{*}=\gamma(1-\delta) y_{1}
\end{aligned}
$$

Since $B<\delta$ when $\gamma>0$ we see that for a range of positive $z_{1}$ it is in fact optimal to spend the entire amount of pocket cash $x_{1}$. Note also that when $\gamma=0$, so there is no self-control problem, so $z_{1}^{*}=0:$ it is never optimal to spend all of the increment to wealth.

The above establishes

Theorem 2: If $z_{1}<z_{1}^{*}$, overall utility is

$$
\log \left(x_{1}+z_{1}\right)+\frac{\delta}{(1-\delta)}\left(\log (1-\delta)+\log \left(R\left(y_{1}-x_{1}\right)\right)+\frac{\delta}{1-\delta} \log (R \delta)\right)
$$

If $z_{1}>z^{*}$ utility is

$$
\begin{aligned}
& (1+\gamma) \log \left(\frac{(1-\delta)(1-\gamma)}{1+\gamma(1-\delta)}\left(y_{1}+z_{1}\right)\right)-\gamma \log \left(x_{1}+z_{1}\right) \\
& +\frac{\delta}{(1-\delta)}\left(\log (1-\delta)+\log \left(\frac{R \delta}{1+\gamma(1-\delta)}\left(y_{1}+z_{1}\right)\right)+\frac{\delta}{1-\delta} \log (R \delta)\right)
\end{aligned}
$$

To analyze risk aversion, imagine that $\tilde{z}_{1}=\bar{z}+\sigma \varepsilon_{1}$, where $\varepsilon_{1}$ has zero mean and unit variance, and suppose that $\sigma$ is very small. Now consider the usual conceptual experiment of comparing a lottery with it certainty equivalent. For $\bar{z}<z^{*}$ overall payoff is given by (6). Thus relative risk aversion is constant and equal to $\rho$, where wealth is $w=x_{1}+\bar{z}_{1}$ so risk is measured relative to pocket cash. On the other hand, for $\bar{z}>z^{*}$, the utility function (7) is the difference between two others, one of which exhibits constant relative risk aversion relative to wealth $y_{1}+\bar{z}$, the other of which exhibits constant risk aversion relative to pocket cash $x_{1}+\bar{z}$. When $\gamma$ is small, the former 
dominates, and to a good approximation for large gambles risk aversion is relative to wealth, while for small gambles it is relative to pocket cash. ${ }^{18}$

We can see this effect graphically in the case of Rabin's [2000] paradox of risk aversion in the small and in the large:

"Suppose we knew a risk-averse person turns down 50-50 lose \$100/gain \$105 bets for any lifetime wealth level less than $\$ 350,000$, but knew nothing about the degree of her risk aversion for wealth levels above $\$ 350,000$. Then we know that from an initial wealth level of $\$ 340,000$ the person will turn down a 50-50 bet of losing $\$ 4,000$ and gaining $\$ 635,670 . "$

The point being of course that many people will turn down the small bet, but no one would turn down the second.

We can easily explain these facts in our model using logarithmic utility. The first bet is most sensibly interpreted as a pocket cash gamble; the experiments with real monetary choices in which subjects exhibit similar degrees of risk aversion certainly are. Moreover, if the agent is not carrying $\$ 100$ in cash, then there may be a transaction cost in the loss state reflecting the necessity of finding a cash machine or bank.

The easiest calculations are for the case where the gain $\$ 105$ is smaller than the threshold $z^{*}$. In this case, logarithmic utility requires the rejection of the gamble if pocket cash $x_{1}$ is $\$ 2100$ or less. That is, $.5 \log (2100-100)+.5 \log (2100+105) \approx \log (2100)$. In order for $\$ 105$ to be smaller than the threshold $z^{*}$, we require $\gamma \geq 105 / x_{1}$, so if $x_{1}=2100$ is pocket cash, we need a $\gamma>.05$, while for ${ }^{19} x_{1}=300, \gamma$ must be of at least 0.35 . However, the conclusion that the gamble should be rejected also applies in some cases where the favorable state is well over the threshold. For example, if pocket cash is $\$ 300, \gamma=0.05$, and wealth is $\$ 300,000$, then the favorable state of $\$ 105$ will be well over the threshold is $\$ 15$, but a computation shows that the gamble should be rejected, and in fact it is not close to the

\footnotetext{
${ }^{18}$ The former also dominates as $\delta$ approaches $1 / R^{1-\rho}$, which is relevant when $\rho \leq 1$.

${ }^{19}$ The usual daily limit in the U.S. for ATM withdrawals is $\$ 300$.
} 
margin. ${ }^{20}$ Indeed, the disutility of the $\$ 100$ loss relative to pocket cash of $\$ 300$ is so large that even a very flat utility for gains is not enough to offset it. Even if we bound the utility of gains by replacing the logarithmic utility with its tangent above $\$ 300$, not only should this gamble be rejected, but even a gamble of lose $\$ 100$, win $\$ 110$ should be rejected.

Turning to the large stakes gamble, unless pocket cash is at least $\$ 4,000$, the second gamble must be for bank cash; for bank cash, the relevant parameter is wealth, not pocket cash. It is easy to check that if wealth is at least $\$ 4,026$, then the second gamble will always be accepted. So, for example, an individual with pocket cash of $\$ 2100$, $\gamma=0.05$ and wealth of more than $\$ 4,026$ will reject the small gamble and take the large one, as will an individual with pocket cash of $\$ 300, \gamma=0.05$ and wealth equal to the rather more plausible $\$ 300,000$.

We should point out that other models can yield these results. In particular, in this specific case, the hyperbolic discounting model yields the identical prediction about bank savings in the first period and second periods, and thus about the response to unanticipated cash shock. To see this, note that our model of response to an unanticipated shock is to maximize the utility function

$$
(1+\gamma) \log c_{1}-\gamma \log \left(x_{1}+z_{1}\right)+\delta U_{2}\left(R\left(y_{1}+z_{1}-c_{1}\right)\right)
$$

Denoting the hyperbolic discount factor by $\beta$, the hyperbolic discounting model says that the response to an unanticipated shock is to maximize the utility function

$$
\log c_{1}+\delta \beta U_{2}\left(R\left(y_{1}+z_{1}-c_{1}\right)\right.
$$

In both cases, the utility function $U_{2}$ is the utility function derived by solving the unconstrained problem, which is the same in the two cases and equal to the utility function of an agent without self-control problems $\left(\beta=1\right.$ or $\gamma=0$.) Since $x_{1}+z_{1}$ is not a decision variable at this "nightclub" stage of the problem, we see that if

\footnotetext{
20 The relationship between pocket cash and wealth depends on $\delta$ and hence on the period length; the relationship is $\delta=1-\left(x_{1} / y_{1}\right)$, so for example, if pocket cash is $\$ 300$ and wealth $\$ 300,000$, then the interest rate corresponding to $\delta$ is $1 / 1000$, or if the annual rate is $10 \%$, the period between bank visits is 3 4 days.
} 
$\beta=1 /(1+\gamma)$ the two objective functions differ only by a linear transformation, and so necessarily yield the same preferences over lotteries at the nightclub stage,

The analysis so far has supposed that cash is only available at the banking stage. If the agent, when banking, anticipates the availability of $\$ 300$ from an ATM during the nightclub stage, it is optimal to reduce pocket cash by this amount. Of course if the goal is to have pocket cash less than $\$ 300$, then self-restraint will be necessary in the presence of cash machines. Note that this explains why we find cash machines where impulse purchases are possible: where lottery tickets are sold, for example. In equilibrium, few if any additional overall sales are induced by the presence of these machines, since their presence is anticipated, but of course the competitor who fails to have one will have few sales. So one consequence of the dual self-model is that we may see an inefficiently great number of cash machines. ${ }^{21}$

Credit cards and checks also pose complications in applying the theory, as for many people the future consequences of using credit cards and checks can be significantly different than the expenditure of cash. That is, it is one thing to withdraw the usual amount of money from the bank, spend it all on the nightclub and skip lunch the next day. It is something else to use a credit card at the nightclub, which, in addition to the reduction of utility from lower future consumption, may result also in angry future recriminations with one's spouse, or in the case of college students, with the parents who pay the credit card bills. So for many people it is optimal to exercise a greater degree of self-control with respect to non-anonymous expenditures such as checks and credit cards, than it is with anonymous expenditures such as cash. This conclusion is consistent with the finding of Wertenbroch, Soman, and Nunes [2002] that individuals who are purchasing a good for immediate enjoyment have a greater propensity to pay by cash, check or debit card than by credit card.

The implications of the theory for experiments are ambiguous and complicated. On the one hand the theory explains why we see substantial risk aversion in the laboratory. On the other hand the theory also predicts a high degree of idiosyncrasy in

${ }^{21}$ Of course businesses engage in a variety of methods to induce impulse purchasing, for example a car salesman's offer to let the purchaser drive away in the car right now. 
that risk aversion. It will depend, for example, on such factors as how much cash the subjects are carrying with them, the convenience of nearby cash machines and the like.

Finally, we should point out that even without a self-control problem, fear of theft can also lead agents to impose binding constraints on their ability to draw against wealth in nightclub periods, and so predicts that unanticipated losses must be absorbed from consumption. This fear-of-theft model predicts that unanticipated gains will be treated the same regardless of whether they are received in cash or in the bank, while the self-control model does not. It is true that the treatment of losses that is relatively more important for resolving the paradox of high risk aversion for small stakes gambles, but for choices between gambles that have only gains, (the usual laboratory case) the "fear of theft" model predicts little risk aversion, where the dual self and hyperbolic discounting model predict that risk aversion will continue to be substantial.

\section{Procrastination and Delay}

We can also use the dual-self model to study procrastination and delay. Consider the following model: Every period $t=1,2, \ldots$ the short-run self must either take an action or wait. Waiting allows the self to enjoy a leisure activity that yields a stochastic amount of utility $x_{t}$, whose value is known at the start of that period; think for example that the leisure activity is playing outside and its utility depends on the weather. We suppose that the $x_{t}$ are i.i.d. with fixed and known cumulative distribution function $P$ and associated density $p$ on the interval $[\underline{x}, \bar{x}]$. Taking the action ends the game, and results in a flow of utility $v$ beginning next period, and so gives a present value of

$$
\frac{\delta}{1-\delta} v \equiv \delta V
$$

If the agent waits, the problem repeats in the next period.

Except for the use of the dual-self model, this model is very similar to that of O'Donoghue and Rabin [2001], who consider hyperbolic preferences. We compare the models after deriving our conclusions.

Because the current value of $x$ has a monotone effect on the payoff to waiting, and no effect on the payoff to doing it now, the optimal solution is a cutoff rule: If

$x \geq x^{*}$ then wait, and if $x<x^{*}$ take the action. The maximum utility that the agent can 
attain in any period is $x_{t}$, which is the payoff to waiting, while doing it now requires foregoing $x_{t}$. Hence waiting incurs no self-control cost, and acting has self-control cost of $\gamma x_{t}$.

If we let $W(x, \gamma)$ denote the value starting tomorrow when using cut-off $x$, and let $W^{*}=W\left(x^{*}(\gamma), \gamma\right)$ denote the value when using the optimal cutoff $x^{*}(\gamma)$, the value of waiting today when the leisure activity is worth $x$ is

$$
x+\delta W^{*},
$$

and the value of acting is

$$
-\gamma x+\delta V
$$

The expected present value tomorrow if the action is not taken today and cutoff rule $x$ is used in the future is then given by

$$
W\left(x^{*}, \gamma\right)=P\left(x^{*}\right)\left(-\gamma E\left(x \mid x<x^{*}\right)+\delta V\right)+\left(1-P\left(x^{*}\right)\right)\left(E\left(x \mid x>x^{*}\right)+\delta W^{*}\right),
$$

so that

$$
W^{*}=\frac{P\left(x^{*}\right)\left(-\gamma E\left(x \mid x<x^{*}\right)+\delta V\right)+\left(1-P\left(x^{*}\right)\right) E\left(x \mid x>x^{*}\right)}{1-\delta+\delta P\left(x^{*}\right)}
$$

Let $\mu=E x$. To avoid uninteresting cases, we assume that present value of acting is greater than the present value of waiting now and forever in the future, when today's opportunity cost is the lowest possible:

$\delta V>\underline{x}+\frac{\delta \mu}{1-\delta}$. 
When the reverse inequality holds, an agent without self-control costs will never act. We also assume that $\delta v<\bar{x}$, so that an agent without no cost of self-control would choose to delay when $x$ is close to $\bar{x}$.

Theorem $3 \quad$ (i) $\underline{x} \leq x^{*}<\bar{x}$; if $\delta v>\delta \mu+(1-\delta)(1+\gamma) \underline{x}$ then $x^{*}>\underline{x}$.

(ii) When $\underline{x}<x^{*}<\bar{x}, \frac{d x^{*}}{d \gamma}<0$, so expected waiting time is increasing in the cost of self control.

Proof: (i) Suppose that the optimum is at $x^{*}=\bar{x}$, and that $x_{t}=\bar{x}$. Then doing it now gives payoff of $\delta V-\gamma \bar{x}$, while waiting one period and then conforming to the presumed optimal rule gives $\bar{x}+\delta^{2} V-\delta \gamma \mu$, because the agent is certain to act next period. Thus waiting is optimal unless $\delta(1-\delta) V \geq \bar{x}+\gamma(\bar{x}-\delta \mu)$, but this contradicts $\delta v<\bar{x}$. Now suppose $x^{*}=\underline{x}$ and $x_{t}=\underline{x}$. Conforming to the strategy yields payoff $\underline{x}+\delta \mu /(1-\delta)$, while acting yields

$$
\delta V-\gamma \underline{x}=\frac{\delta v}{(1-\delta)}-\gamma \underline{x}
$$

so acting is better if $(1-\delta) \underline{x}+\delta \mu<\delta v-(1-\delta) \gamma \underline{x}$ or $\delta v>(1-\delta)(1+\gamma) \underline{x}+\delta \mu$.

(ii) If the optimal cutoff $x^{*}$ is in the interior of $[\underline{x}, \bar{x}]$, optimality implies that the agent is indifferent between waiting and acting when $x=x^{*}$. Thus $-\gamma x^{*}+\delta V=x^{*}+\delta W\left(x^{*}, \gamma\right)$, so

$$
x^{*}(1+\gamma)=\delta V-\delta W\left(x^{*}, \gamma\right)
$$

Because $x^{*}$ maximizes steady state payoff, $\left.W_{x}\right|_{x=x^{*}}=0$, Thus 


$$
(1+\gamma) \frac{d x^{*}}{d \gamma}=-x^{*}-W_{\gamma}\left(x^{*}(\gamma), \gamma\right)
$$

SO

$$
\frac{d x^{*}}{d \gamma}<0 \text { if } x^{*}>-W_{\gamma}\left(x^{*}(\gamma), \gamma\right)
$$

Now from (8),

$$
\begin{gathered}
-W_{\gamma}=\frac{P\left(x^{*}\right)}{1-\delta+\delta P\left(x^{*}\right)} E\left(x \mid x<x^{*}\right) \\
<\frac{P\left(x^{*}\right)}{(1-\delta) 1+\delta P\left(x^{*}\right)} x^{*}<x^{*} .
\end{gathered},
$$

So $\frac{d x^{*}}{d \gamma}<0$.

Let $x_{n}$ (for "naïve") be the cutoff the agent would use if forced to make a choice between stopping now and never stopping. Then

$$
(1+\gamma) x_{v}=\delta V-\frac{\delta \mu}{1-\delta}
$$

When the optimal rule is interior, the agent eventually does choose to stop, and so the continuation value $W^{*}$ exceeds $\frac{\delta \mu}{1-\delta}$, and equation (9) shows that $x^{*}<x_{n}$. This shows that the standard "option value of waiting" consideration carries over to the dual-self model. 
In independent work, Miao [2004] applies a dual-self model to a very similar problem. He considers the cases of immediate costs and future benefits, as here; immediate benefits and future rewards, where the temptation is to act to soon, and immediate costs and immediate rewards. His model differs in that the reward is stochastic and the cost is fixed.

O'Donoghue and Rabin [2001] analyze the implications of hyperbolic discounting in a very similar (and in particular, stationary) stopping time problem. They say that the agent "procrastinates" if he never acts even though there is an action that is worth doing given his hyperbolic discounting of future returns. They also show that their model typically has multiple equilibria; the equilibria are cyclic, with intervals of length $T$ between "action dates." We view these cyclic equilibria as artificial and unappealing. Moreover, they restrict attention to equilibria that are limits of equilibria in the finite horizon; this restriction relies on long chains of backwards induction and is not robust, to even small payoff uncertainty as shown by Fudenberg, Kreps, and Levine [1988].. Despite the presence of multiple equilibria, O'Donoghue and Rabin can show that sophisticated agents (that is those who know their own hyperbolic parameter $\beta$ ) never procrastinate, although they may postpone acting for a few periods.

DellaVigna and Malmendier [2003] report some calibrations of the O'DonoghueRabin model to data on delay in canceling health club memberships, which they attribute to a combination of hyperbolic preferences and "lack of sophistication," meaning that consumers misperceive their own hyperbolic parameter and thus incorrectly forecast their health club usage. ${ }^{22}$ Our model suggests several qualifications to their analysis. First of all, as is standard in models of timing, it is not in general optimal for the agent to act whenever he is indifferent between acting now or not at all, as there is an "option value" in waiting. ${ }^{23}$ Second, while there is some evidence that agents do not have perfect

\footnotetext{
${ }^{22}$ Sophisticated, "low $\beta$ " agents who have correct perceptions about the costs and benefits of the club would correctly forecast that they would rarely attend but take a long time to cancel, while agents who misperceive their $\beta$ would expect to exercise a lot. DellaVigna and Malmendier also show that agents choose monthly or annual plans with no per-visit charge when it would be cheaper to pay per visit. The use of prepayment as a commitment device is a consequence of both the hyperbolic and dual-self models.

23 This factor is also present in the O'Donoghue-Rabin model, but the discussion of cancellation in DellaVigna and Malmendier [2003] seems to use a deterministic specification for the costs of cancellation. Also, the calibration measures cancellation lag by the number of full months between the last attendance and contract termination for users who hold a monthly contract at the time of termination. This is a
} 
knowledge about themselves, we expect them to have more information about things that they have had more chances to observe. ${ }^{24}$ Thus it seems natural to assume that the misperceptions about the short-run disutility and long-run benefits of going to the health club are larger than misperceptions about their own impulsiveness, and of course these former misperceptions can also explain the excessive delay.

We can also compare the dual-self model to the deterministic, finite horizon model of O'Donoghue and Rabin [1999], which allows non-stationary costs. In their Example 1, there are 4 periods to do a report, with costs $3,5,8,13$, and value $v$. Applying our model to this problem, we see that in the final period there is no selfcontrol problem, so the payoff to acting is $v-13$. In the next to last period the short-run self gets 0 from waiting, -8 from acting, so the utility if doing now is $v-8(1+\gamma)$; in the previous periods the payoffs are $v-5(1+\gamma), v-3(1+\gamma)$ respectively. Thus the solution in our model is: if $\gamma<10 / 3$ do it at the start, else do it at the end, and never do in intermediate periods. This is different than their solution, even for the case of a sophisticated agent, as the sophisticated agent acts in the second and in the fourth periods. Basically this equilibrium corresponds to one of the unappealing cyclic equilibria in their infinite horizon model; with an odd number of periods, the equilibrium is for the sophisticated agent to act in the first, third and fifth periods. ${ }^{25}$

\section{Cognitive Load and Self Control}

Shiv and Fedorikhin [1999] report on the following experiment. Subjects were asked to memorize either a two- or a seven-digit number, and then walk to a table with a choice of two deserts, namely chocolate cake and fruit salad. Subjects would then pick a

conservative estimate if the customer knows that she will not attend in the future by the end of the month that included the customer's last visit, but otherwise may exaggerate the amount of delay.

${ }^{24}$ Bodner and Prelec [2003] and Benabou and Tirole [2004] build on the idea of imperfect self-knowledge to develop models of "self-signalling' that they use to explain the use of "personal rules." These models assume that the agent is uncertain of only one thing. In Benabou and Tirole [2004], for example, the agent knows the distribution of costs but does not know his hyperbolic parameter $\beta$. Both Bodner and Prelec [2003] and Benabou and Tirole [2004] analyze Bayesian equilibria of their models, which raises the question of whether a plausible non-equilibrium learning process would lead agents to learn the strategy of their "other selves" without learning the underlying value of $\beta$. Dekel et al [2004] analyze this issue in games between multiple agents; their results show that when $\beta$ if fixed over time, assumptions that allow the agent not to learn $\beta$ are typically too weak to justify restricting attention to equilibrium.

25 The multiple-selves version of the delay game is continuous at infinity, so from Fudenberg and Levine [1983] every limit of finite-horizon subgame-perfect equilibria is a subgame-perfect equilibrium in the infinite horizon. 
ticket for one of the deserts, and go to report both the number and their choice in a second room. In one treatment, actual samples of the deserts were on the table, and in a second treatment, the deserts were represented by photographs. The authors' hypothesize that subjects will face a self-control problem with respect to the cake, in the sense that it will have higher emotional appeal but be less desirable from the "cognitive" viewpoint; that the subject's reaction is more likely to be determined by the emotional ("affective") reaction when cognitive resources are constrained by the need to remember the longer number, and that this effect will be greater when faced with the actual deserts than with their pictures. ${ }^{26}$ The experimental results confirm these predictions. Specifically, when faced with the real deserts, subjects who were asked to remember the seven-digit number chose cake $63 \%$ of the time, while subjects given the easier two-digit number chose cake $41 \%$ of the time, and this difference was statistically significant. In contrast, when faced with the pictures of the deserts, the choices were $45 \%$ and $42 \%$ respectively, and the difference was not significant.

The finding that the increasing the cognitive load increases responsiveness to temptation can be easily captured in our model by the assumption that the cost of selfcontrol is higher when the long-run self - which we identify with cognitive processing has other demands on its resources. Here are two ways of formalizing this. First, we can assume that the cognitive center has a fixed amount $D$ of cognitive resources, and that the resources required for self-control are proportional to the short-run foregone utility. Then when other cognitive tasks consume $d$ resources, the utility of the short-run self can be reduced by at most $D-d$; greater self-control is simply infeasible. This model violates our Assumption 2, and since it corresponds to infinite costs for certain actions it also violates the linearity condition assumption 5 . and so violates the Gul-Pesendorfer axioms.

Alternatively, we can suppose that the cognitive center does not face a fixed resource constraint, but instead has increasing marginal cost, again abandoning assumption 5. Let $d$ be the resources required for cognitive tasks such as short-term memory, let $s$ be the resources required for self control, and let $g(d+s)$ be the disutility of the resources used in the two tasks, with the resources required for self-control

\footnotetext{
${ }^{26}$ They base this last hypothesis on the work of Lowenstein [1996].
} 
proportional to the foregone utility, and thus equal to $\gamma g$. This reduces to our base model if $g(d)=\gamma d$, but we now want to assume that cost is convex, that is, $g^{\prime}>0$ and $g^{\prime \prime}>0 .^{27}$

Let $u^{c}$ and $u^{f}$ be the short-run utilities of chocolate and fruit respectively, with $u^{c}>u^{f}$. Then $s($ fruit $)=u^{c}-u^{f}$, and the overall utility functions are $U($ chocolate $)=u^{c}-g(d)$ and $U($ fruit $)=u^{f}-g\left(d+u^{c}-u^{f}\right)$. This implies that increases in $d$ make chocolate comparatively more attractive.

In the hyperbolic model, there is a sequence of far-sighted individuals with timeinconsistent preferences parameterized by $\beta$, so the most obvious way to explain the Shiv and Fedorikhin result is to assume that $\beta$ decreases when the cognitive center has other tasks. This is roughly analogous to our proposal, but to us it seems more natural and direct to assume that cognitive load uses up self-control resources.

The effect of substituting photographs for the actual deserts shows the importance of cues and framing: The evidence supports Lowenstein's theory that vividness influences the effect of temptation. This raises the conjecture that the use of "rules of thumb" like "only have sweets at dinner" aids self-control buy reducing the vividness of the temptation; confirming this would require comparing brain scans of agents who use these rules to other agents who do not.

\section{Conclusion and Discussion}

Our resolution of the Rabin paradox shows how the dual self model captures "framing" effects. In our model it makes a difference whether an unanticipated payment is received on the floor of a casino or the lobby of a bank. Cues are obviously the key to understanding framing. The dual self theory implies that it is the attention span of the short-run self that is relevant for determining what constitutes a "situation" - the most difficult modeling issue in confronting these types of issues. This suggests that one might be able to use experimental and physiological data to determine what the relevant frames are. The dual self theory would then enable us to paste information about the motivation of the myopic self into the broader context in which real decision making takes place.

\footnotetext{
27 The model with convex costs does not satisfy assumption 5, so it does not satisfy the Gul-Pesendorfer axioms. It would be interesting to know how to relax those axioms to include this specification.
} 
Most existing work on cues, such as Laibson [2001] and Bernheim and Rangel [2004], abstracts away from self-control costs. Laibson analyzes a "rational addiction" model- that is, a model with a single, fully rational, agent - in a setting where there are two cues, namely "green lights " and "red lights," and the utility from engaging in the addictive utility when a given light is on depends on one's past behavior under that particular light. Because it is based on rational choice, the model has a unique equilibrium, but that equilibrium has four steady states: never indulge, always indulge, indulge iff green, and indulge iff red. This shows how the agent's experience can determine the importance of the cues, but does not allow the agent to have a preference for self-control. Bernheim and Rangel [2004] consider an addiction model where the agent can sometimes enters a "hot mode" in which he consumes the addictive good whether or not his "cool self" wants him to. The probability that this occurs depends on the observed cues, while which cues trigger the hot mode in turn depends on the agent's past frequency of use and also on whether the cool self chooses to expend resources on avoiding the cues. This model, like ours, captures a value for self-control while avoiding the multiple equilibria of a multiple-selves model, but it differs in a number of respects. Most notably, in their model self control is costless in the cool mode and infinitely costly in the hot one. In our model the agent is in a "hot mode" whenever his actions have shortterm consequences, but even in hot mode the long-run self can exert self control. The fact the self-control is always possible, albeit costly, is what underlies our finding that the agent responds differently to small versus large gambles in our banking model: a sufficiently large windfall will trigger self control and the long-term perspective.

We focus on the case of "sophisticated" agents who are aware of their own selfcontrol costs. Many papers on self-control problems consider the case of "naïve" agents, who have a current self-control problem but incorrectly forecast that they will not have such problems in the future. For example, O'Donoghue and Lowenstein [2004] consider an extension similar to allowing agents to misperceive the future value of $\gamma$. O'Donoghue and Lowenstein also point out that that if the current long-run self has correct expectations but does not care about future costs of self control, the decision 
making process becomes a game between the long-run selves, and the result is equivalent to the usual quasi-hyperbolic discounting. ${ }^{28}$

A more complicated extension is allowing for the preferences of the short-run selves to respond in some way to future consequences. The related work of Benhabib and Bisin [2004] does allow for one form of this responsiveness. They consider a consumption-savings model in which exercising self-control is a 0-1 and costly decision, made by a "cognitive control center" that corresponds to the long-run self in our model. Temptation is stochastic, and its strength is determined by an exogenous i.i.d. sequence of cues, so that costly self-control is only used when giving in to temptation is sufficiently costly. The equilibrium again corresponds to the solution of an optimization problem by the long-run self, who takes the behavior rule of the affective self as given. In contrast to our own paper and other related work, the behavior of the "affective self" the mental unit that is susceptible to temptation- is not required to be myopic. Instead, the affective self's strategy can depend on expectations of future play, but it must be independent of the distribution of temptations and the cost of self control. As an example, they suggest the case where the behavior of the affective self is a Markov-perfect equilibrium of the game where self-control is impossible. If the actual outcome is that the cognitive center does sometimes exercise self-control, this raises the question of how the adaptive selves would come to learn the equilibrium play of the wrong game. ${ }^{29}$

Instead of following the expectations-based approach, we would like to model the long-run self having "taught" the short-run self to attach positive affective weight to certain variables that have long-run consequences, as in the learning of cues. Here the use of stimulus-response models of learning may play an important role. The standard forms of these models seem to be a poor fit for many aspects of human cognition. ${ }^{30}$ For example, faced with no observations people will respond differently depending on their

\footnotetext{
28 They also use their model to explain non-linear probability weighting in the assessment of risks

${ }^{29}$ It is of course possible that either the short-run or long-run self or both may misperceive the future. However, learning makes it less likely that these misperceptions will involve frequently experienced variables such as $\gamma$, or more broadly, one's own ability to exercise self-control. Moreover, when little learning occurs, it is not clear why one should expect to see equilibrium play.

${ }^{30}$ By the "standard model" here we mean that reinforcements are applied directly to actions. Stimulusresponse dynamics can be defined on much larger spaces of sequences of actions, hypothetical reinforcements, etc.; at that level of generality they encompass a much larger set of phenomena.
} 
prior knowledge of a situation. ${ }^{31}$ More strikingly, we know that people can learn by "figuring things out" without any external stimulus at all. However, these cognitive activities can be sensibly regarded as aspects of the long-run self, while it makes sense to model the expectations of the short-run self as arising from process of stimulus-response learning that depends solely on the past history, and does not involve forward-looking expectations. We would then theorize, based on introspection and casual empiricism, that the long-run self can train the short-run self by manipulating this stimulus-response learning.

\footnotetext{
${ }^{31}$ For a particularly striking experiment where learning takes place in the absence of feedback, see Roberto Weber [2003].
} 


\section{References}

Benabou, R. and M. Pycia [2002] "Dynamic Inconsistency and Self-Control," Economics Letters 77, 419-424

Benabou, R. and J. Tirole [2004]: "Willpower and Personal Rules, Journal of Political Economy, 112, 848-887.

Benahib, J. and A. Bisin [2004] "Modeling Internal Commitment Mechanisms and SelfControl: A Neuroeconomics Approach to Consumption-Saving Decisions," mimeo.

Bernheim, B.D. and A. Rangel [2004] "Addiction and Cue-Triggered Decision Processes," mineo, forthcoming in the American Economic Review

Bodner, R. and D. Prelec [2003]: “The Diagnostic Value of Actions in a Self-Signaling Model, in I. Brocas and J. Carillo, (eds.), The Psychology of Economic Decisions, Vol. 1, Oxford University Press, Oxford.

Camerer, C., G. Lowenstein, and D. Prelec [2000]: "Why Economists Need Brains," http://www.hss.clatech.edu/ camerer/scanjecon11final.doc

Dekel, E., D. Fudenberg, and D. K. Levine [2004]: "Learning to Play Bayesian Games," Games and Economic Behavior, 46: 282-303.

DellaVigna, S. and U. Malmendier, [2003]: "Overestimating Self-Control: Evidence From the Health Club Industry,” Stanford GSB Research Paper 1800.

DellaVigna, S. and U. Malmendier [2003]: "Contract Design and Self Control: Theory and Evidence," http://emlab.berkeley.edu/users/sdellavi/wp/self_control_jan04.pdf.

Fudenberg, D., D. Kreps and D. K. Levine [1988]: “On the Robustness of Equilibrium Refinements," Journal of Economic Theory, 44: 354-380

Fudenberg, D. and D. K. Levine [1983]: "Subgame-Perfect Equilibria of Finite- and Infinite-Horizon Games," Journal of Economic Theory, 31: 251-258.

Fudenberg, D. and D. K. Levine [1998]: Theory of Learning in Games, MIT Press, Cambridge MA.

Gul, F. and W. Pesendorfer [2001]: "Temptation and Self Control," Econometrica, 69: 1403-1436.

Gul, F. and W. Pesendorfer [2002]: "Self Control and the Theory of Consumption," forthcoming, Econometrica. 
Gul, F. and W. Pesendorfer [2004]: "Self Control, Revealed Preference and Consumption Choice," forthcoming, Review of Economic Dynamics.

Harris, C. and D. Laibson [2004]: "Instantaneous Gratification,” mimeo.

Haruno, M. , T. Kuroda, K. Doya, K. Toyama, M. Kimura, K. Samejima, H. Imamizu, and M. Kawato [2004]: "A Neural Correlate of Reward-Based Behavioral Learning in Caudate Nucleus: A Functional Magnetic Resonance Imaging Study of a Stochastic Decision Task," The Journal of Neuroscience, 24:1660-1665.

Krusell, P. and T. Smith. [2000]: "Consumption-Savings Decisions with QuasiGeometric Discounting," Econometrica.

Laibson, D. [1997]. "Golden eggs and hyperbolic discounting." Quarterly Journal of Economics, 112, 443-477.

Laibson, D. [2001] "A Cue-Theory of Consumption," Quarertly Journal of Economics, 116, 81-120.

Lowenstein. G. [1996] "Out of Control: Visceral Influences on Behavior," Organizational Behavior and Human Decision Process, 65,272-292.

Lowenstein, G. and T. O'Donoghue [2004] "Affective and Deliberative Processes in Economic Behavior," mimeo,

McItnosh, D. [1969] The Foundations of Human Society, U. Chicago Press, Chicago

McClure, S.M., Laibson, D.I., Loewenstein, G., Cohen, J.D. [2004]: "Separate neural systems value immediate and delayed monetary rewards," Science, forthcoming

Miao. Jianjun [2004] “Option Exerise with Temptation,” mimeo.

O’Doherty, J. [2004] "Reward Representations and Reward-Related Learning in the Human Brain: Insights from Neuroimaging," Current Opnion in Neurobiology, 14:769-776.

O'Donoghue, T. and M. Rabin [1999]: "Doing It Now or Later," American Economic Review 89, 103-124.

O'Donoghue, T. and M. Rabin [2001]: "Choice and Procrastination" Quarterly Journal of Economics, 121-160.

Platt , M.L., and P. Glimcher [1999] "Neural correlates of decision variables in parietal cortex." Nature 400:233-238.

Prelec, D. [2004] "Decreasing impatience: A criterion for non-stationary time preference and 'hyperbolic' discounting," mimeo. 
Rabin, M., [2000]: "Risk Aversion and Expected-Utility Theory: A Calibration Theorem," Econometrica, 1281-1292.

Shiv, B. and A. Fedorikhin [1999]: "Heart and Mind in Conflict: The Interplay of Affect and Cognition in Consumer Decision Making," Journal of Consumer Research, 26, 72-89.

Strotz, R. H. [1955]: Myopia and inconsistency in dynamic utility maximization. Review of Economic Studies, 23, 165-180.

Thaler, R. and H.M. Shefrin [1981]: “An Economic Theory of Self-Control,” Journal of Political Economy, 89, 392-406.

Weber, Roberto A. [2003]: "Learning and Transfer of Learning with No Feedback: An Experimental Test Across Games," Carnegie Mellon University.

Wertenbroch, K., D. Soman, and J. Nunes [2002]: "Debt Aversion and Self-Control: Consumer Self-Management of Liquidity Constraints." Mimeo. 


\section{Appendix}

I. We first give a result for general per-period utility functions in the simple savings model. Consider the problem of maximizing

$$
U(\vec{a})=\sum_{t=1}^{\infty} \delta^{t-1}\left[(1+\gamma) u\left(\left(1-a_{t}\right) y_{t}\right)-\gamma u\left(y_{t}\right)\right]
$$

over all feasible plans $\vec{a}$, i.e. plans that satisfy $a_{t} \in[0,1]$ and the wealth equation $y_{t}=R a_{t-1} y_{t-1}$. We suppose that $u$ is non-decreasing and continuous on $(0, \infty)$; we do not require continuity on $[0, \infty)$ because we want to allow for the logarithmic case where $u(0)=\lim _{c \rightarrow 0} u(c)=-\infty$. Let $\bar{U}$ be the supremum in this problem.

Proposition A.1: Suppose $R>1$ and

$$
\sum_{t=1}^{\infty} \delta^{t-1} u\left(y_{0} R^{t}\right)<\infty
$$

Then: (i) For any feasible plan the sum defining $U$ has a well defined value in the sense that either the sum converges absolutely or converges to $-\infty$.

(ii) The supremum $\bar{U}$ of the feasible values satisfies $-\infty<\bar{U}<\infty$

(iii) If feasible $\vec{a}^{n} \rightarrow \vec{a}^{*}$ in the product topology then $\vec{a}^{*}$ is feasible. If in addition $U\left(\vec{a}^{n}\right) \rightarrow \bar{U}$ then $U\left(\vec{a}^{*}\right)=\bar{U}$.

(iv) An optimal plan exists. That is, there is a feasible plan that attains $\bar{U}$.

Proof: (i) For any sequence $(\vec{a}, \vec{y})$ with $y_{t}=R a_{t-1} y_{t-1}$, let

$$
\chi_{+}(x)= \begin{cases}x & x>0 \\ 0 & x \leq 0\end{cases}
$$

and $\chi_{-}(x)=-\chi_{+}(-x)$. We can write any finite sum as the sum of negative and positive parts. 


$$
\begin{aligned}
& \sum_{t=1}^{T} \delta^{t-1}\left[(1+\gamma) u\left(\left(1-a_{t}\right) y_{t}\right)-\gamma u\left(y_{t}\right)\right]= \\
& \sum_{t=1}^{T} \delta^{t-1} \chi_{+}\left[(1+\gamma) u\left(\left(1-a_{t}\right) y_{t}\right)-\gamma u\left(y_{t}\right)\right]+ \\
& \sum_{t=1}^{T} \delta^{t-1} \chi_{-}\left[(1+\gamma) u\left(\left(1-a_{t}\right) y_{t}\right)-\gamma u\left(y_{t}\right)\right]
\end{aligned}
$$

The positive part of the sum is summable from (A.1), since

$$
(1+\gamma) u\left(\left(1-a_{t}\right) y_{t}\right)-\gamma u\left(y_{t}\right) \leq u\left(\left(1-a_{t}\right) y_{t}\right) \leq u\left(y_{0} R^{t}\right)
$$

The negative part is monotone decreasing in $T$, so it either converges absolutely or converges to $-\infty$. In the former case the entire sum converges absolutely; in the latter case the sum converges to $-\infty$.

(ii) Part (i) already shows that $\bar{U}<\infty$. To see that $\bar{U}>-\infty$, note that it is feasible to set $a_{t}=1 / R$ for all $t$, and that for $R>1$ this plan yields a finite value.

(iii) Consider a sequence of feasible plans $\vec{a}^{n} \rightarrow \vec{a}^{*}$. Because the constraints are period by period and closed, it is clear that $\vec{a}^{*}$ satisfies the constraints, so it is feasible. Now suppose in addition that $U\left(\vec{a}^{n}\right) \rightarrow \bar{U}$. Choose any $\varepsilon>0$ and pick n large enough that $\bar{U}-U\left(\vec{a}^{n}\right)<\varepsilon / 2$. If we now pick $\tau$ such that

$$
\sum_{t=\tau+1}^{\infty} \delta^{t-1}\left|u\left(R^{t} y_{0}\right)\right|<\varepsilon / 2
$$

we know that

$$
\bar{U}-\sum_{t=1}^{\tau} \delta^{t-1}\left[(1+\gamma) u\left(\left(1-a_{t}^{n}\right) y_{t}^{n}\right)-\gamma u\left(y_{t}^{n}\right)\right] \leq \varepsilon
$$

Since $\bar{U}$ is finite, and payoffs in the first $\tau$ periods are bounded above by $u\left(y_{0} R^{t}\right)$, each term in this summation is bounded below (by $\bar{U}-\varepsilon-T u\left(y_{0} R^{t}\right)$ ). Since per-period payoffs are continuous at any $(a, y)$ with $a>0, \vec{a}^{n} \rightarrow \vec{a}^{*}$, and $y_{t}^{n} \rightarrow y_{t}^{*}$, it follows that

$$
\bar{U}-\sum_{t=1}^{\tau} \delta^{t-1}\left[(1+\gamma) u\left(\left(1-a_{t}^{*}\right) y_{t}^{*}\right)-\gamma u\left(y_{t}^{*}\right)\right] \leq \varepsilon
$$


Since this is true for any $\varepsilon>0$ and we know that $U\left(\vec{a}^{*}\right) \leq \bar{U}$, we conclude that $U\left(a^{*}\right)=\bar{U}$.

(iv) Now consider a feasible sequence $\left(\vec{a}^{n}, \vec{y}^{n}\right)$ with $U\left(\vec{a}^{n}\right) \rightarrow \bar{U}$. Each savings rate $a_{t}$ must lie in the compact interval $[0,1]$ and each $y_{t}$ must lie in the compact interval $\left[0, R^{t} y_{0}\right]$, so the sequence $\left(\vec{a}^{n}, \vec{y}^{n}\right)$ has an accumulation point $\left(\vec{a}^{*}, \vec{y}^{*}\right)$ in the product topology. This accumulation point is a maximum by part (iii).

II. Now we specialize to the CRRA utility functions

$$
u(c)=\frac{(c)^{1-\rho}-1}{1-\rho}
$$

and $u(c)=\ln (c)$, which corresponds to the case $\rho=1$. Assuming $\delta<R^{\rho-1}$ implies

$$
\sum_{t=1}^{\infty} \delta^{t-1} u\left(y_{0} R^{t}\right)<\infty
$$

It follows from Proposition A. 1 that an optimum $\vec{a}^{*}$ exists.

Proposition A.2: With CRRA utility a stationary optimum with $a_{t}=a$ exists.

Proof: Suppose that $\vec{a}^{*}$ is an optimal plan. By homogeneity of the objective function, and the fact that plans are defined in terms of savings rates, $\vec{a}^{*}$ is also an optimal plan starting in period 2 (for any initial condition). Note that the plan $\vec{a}^{2}=\left(a_{1}^{*}, a_{1}^{*}, a_{2}^{*}, a_{3}^{*}, \ldots\right)$ yields wealth in period 2 of $a_{1}^{*} R y_{1}$, and let $\bar{U}\left(y_{1}\right)$ denote the maximized utility when starting in the second period with wealth $y_{1}$. Then

$$
U\left(\vec{a}^{2}\right)=(1+\gamma) u\left(\left(1-a_{1}^{*}\right) y_{0}\right)-\gamma u\left(y_{0}\right)+\delta \bar{U}\left(a_{1}^{*} R y_{0}\right)=\bar{U}
$$

where the first equality follows because $\vec{a}^{*}$ is optimal from period 2 on, and the second equality because $\vec{a}^{*}$ is optimal from the first period. Proceeding in this way we can construct sequence of feasible plans $\vec{a}^{n}=\left(a_{1}^{*}, a_{1}^{*}, \ldots, a_{1}^{*}, a_{2}^{*}, a_{3}^{*}, \ldots\right)$ that play $a_{1}^{*}$ for the first $n$ periods such that $U\left(\vec{a}^{n}\right)=U\left(a^{*}\right)=\bar{U}$. Clearly $\vec{a}^{n}$ converges in the product topology to the plan of choosing the fixed savings rate $a_{1}^{*}$. Hence it follow from Proposition A.1 (iii) that this limiting plan is feasible and gives utility $\bar{U}$; that is, it is optimal. 
III. We have shown that it is sufficient to compute the present value utility from a fixed savings rate $a$, and maximize over savings rates. We have present value utility

$$
\begin{aligned}
U & =\frac{y_{0}^{1-\rho}}{1-\rho} \sum_{t=1}^{\infty}\left(\delta(R a)^{1-\rho}\right)^{t-1}\left[(1+\gamma)(1-a)^{1-\rho}-\gamma\right]-\frac{1}{(1-\delta)(1-\rho)} \\
& =\frac{y_{0}^{1-\rho}}{1-\rho} \frac{\left[(1+\gamma)(1-a)^{1-\rho}-\gamma\right]}{1-\delta(R a)^{1-\rho}}-\frac{1}{(1-\delta)(1-\rho)}
\end{aligned}
$$

Since the optimal savings rate cannot be 0 or 1 , we may differentiate with respect to the saving rate to find

$$
\begin{aligned}
& d U / d a= \\
& y_{0}^{1-\rho}(1-a)^{-\rho} \frac{\left[(1+\gamma)(1-a)-\gamma(1-a)^{\rho}\right] \delta R(R a)^{-\rho}-(1+\gamma)\left(1-\delta(R a)^{1-\rho}\right)}{\left(1-\delta(R a)^{1-\rho}\right)^{2}}
\end{aligned}
$$

which gives necessary condition for an optimum ${ }^{32}$

$$
(1+\gamma) a^{\rho}=R^{1-\rho} \delta\left((1+\gamma)-\gamma(1-a)^{\rho}\right)
$$

When $\gamma=0$ we get the usual solution $a^{*}=R^{(1-\rho) / \rho} \delta^{1 / \rho}$. Thus we can rewrite the first order condition as

$$
\left(a / a^{*}\right)^{\rho}=\left((1+\gamma)-\gamma(1-a)^{\rho}\right) /(1+\gamma)
$$

IV: Turning to the simple banking model, utility starting in the second period is the $\gamma=0$ solution

\footnotetext{
${ }^{32}$ We do not know if the first-order condition has a unique solution, except in the logarithmic case.
} 


$$
\begin{aligned}
U_{2}\left(y_{2}\right) & =\frac{y_{2}^{1-\rho}}{1-\rho} \frac{\left(1-a^{*}\right)^{1-\rho}}{1-\delta\left(R a^{*}\right)^{1-\rho}}-\frac{1}{(1-\delta)(1-\rho)} \\
& =\frac{y_{2}^{1-\rho}}{1-\rho} \frac{1}{\left(1-a^{*}\right)^{\rho}}-\frac{1}{(1-\delta)(1-\rho)} \\
& =\frac{y_{2}^{1-\rho}}{1-\rho} \frac{1}{\left(1-\delta^{1 / \rho} R^{(1-\rho) / \rho}\right)^{\rho}}-\frac{1}{(1-\delta)(1-\rho)}
\end{aligned}
$$

The utility of both selves in the first period is

$$
(1+\gamma) \frac{\left(c_{1}\right)^{1-\rho}-1}{1-\rho}-\gamma \frac{\left(x_{1}+z_{1}\right)^{1-\rho}-1}{1-\rho},
$$

and so the overall objective of the long-run self is to maximize

$$
\begin{aligned}
& (1+\gamma) \frac{\left(c_{1}\right)^{1-\rho}-1}{1-\rho}-\gamma \frac{\left(x_{1}+z_{1}\right)^{1-\rho}-1}{1-\rho} \\
& +\frac{\left(R\left(y_{1}+z_{1}-c_{1}\right)\right)^{1-\rho}}{1-\rho} \frac{\delta}{\left(1-\delta^{1 / \rho} R^{(1-\rho) / \rho}\right)^{\rho}}-\frac{\delta}{(1-\delta)(1-\rho)}
\end{aligned}
$$

The first order condition for optimal consumption is

$$
\frac{c_{1}}{R\left(y_{1}+z_{1}-c_{1}\right)}=\frac{(1+\gamma)^{1 / \rho}\left(1-\delta^{1 / \rho} R^{(1-\rho) / \rho}\right)}{(\delta R)^{1 / \rho}} .
$$

If there are one or more solutions that satisfy the constraint $c_{1} \leq x_{1}+z_{1}$ then one of them represents the optimum; otherwise the optimum is to consume all pocket cash, $c_{1}=x_{1}+z_{1}$.

Note that $x_{1}$ is the solution for $\gamma=0$, so it satisfies

$$
\frac{x_{1}}{R\left(y_{1}-x_{1}\right)}=\frac{\left(1-\delta^{1 / \rho} R^{(1-\rho) / \rho}\right)}{R^{1 / \rho}} .
$$

Thus we can write the first order condition as

$$
\frac{c_{1}}{y_{1}+z_{1}-c_{1}}=(1+\gamma)^{1 / \rho} \frac{x_{1}}{y_{1}-x_{1}}
$$


or

$$
\begin{aligned}
c_{1} & =\frac{(1+\gamma)^{1 / \rho} x_{1}}{y_{1}-x_{1}+(1+\gamma)^{1 / \rho} x_{1}}\left(y_{1}+z_{1}\right) \\
& =\frac{1+\left[(1+\gamma)^{1 / \rho}-1\right]}{1+\left[(1+\gamma)^{1 / \rho}-1\right]\left(1-a^{*}\right)}\left(1-a^{*}\right)\left(y_{1}+z_{1}\right) \\
& =B\left(1-a^{*}\right)\left(y_{1}+z_{1}\right)
\end{aligned}
$$

